\section{Cervical cancer}

Poster (C01)

Cervical Cancer

https://doi.org/10.3802/jgo.2021.32.S1.c01

\section{Dosimetric comparison of single $6 \mathrm{MV}$, single $10 \mathrm{MV}$, and mixed $6 \mathrm{MV}$ plus $10 \mathrm{MV}$ energy volumetric modulated arc therapy for cervical cancer}

\author{
Pongpop Tuntapakul,," Pittaya Dankulchai, ${ }^{2}$ \\ Lalida Tuntipumiamorn ${ }^{2}$ \\ 'Maha Vajiralongkorn Thanyaburi Hospital, Thanyaburi, Thailand \\ (tpongpop@gmail.com) \\ ${ }^{2}$ Siriraj Hospital, Bangkok, Thailand
}

Objective: Volumetric Modulated Arc Therapy (VMAT) in the pelvic and groin lymph node irradiation is generally employed with single energy photon. Due to the different depth of target volumes, the mixed energy photon VMAT could produce better dose distribution since the higher dose energy photon penetrates more to deep-seated tumors and the lower dose energy photon better covers superficial targets. This pilot study compares the dosimetric parameters generated from mixed energy $6 \mathrm{MV}$ plus 10MV VMAT plan and single energy of 6MV and 10MVVMAT plan. Methods: Ten cervical cancers with lower one-third vaginal involvement patients were selected. The dose of planning target volume (PTV)-pelvis and PTV-gross lymph node was 45 Gy (PTV-45) and 55 Gy (PTV-55), respectively. VMAT using 6MV, 10MV, and mixed energy $6 \mathrm{MV}$ plus $10 \mathrm{MV}$ were generated with identical plan objectives. Dosimetric parameters of PTV and organ at risks were compared. Results: Almost parameters showed no statistically significant difference among three different energies. For exception, the dose coverage $98 \%$ of PTV- 45 of mixed energy was significantly higher than of 10MV. The dose coverage 98\% of PTV-55 of mixed energy was significantly higher than of 10MV. The Homogeneity Index of PTV45 of $6 \mathrm{MV}$ was significantly better than of 10MV.The mean dose of external genitalia of $6 \mathrm{MV}$ was significantly less than of mixed energy. Conclusion: The mixed energy 6MV plus 10MV VMAT plan in the pelvis with inguinal lymph nodes irradiation for cervical cancer did not show explicit benefit in term of dosimetric outcomes, compared to single energy.

Poster (CO2)

Preinvasive Disease of Cervix, Vulva, and Vagina

https://doi.org/10.3802/jgo.2021.32.S1.C02

\section{Epigenetic and genetic characteristics of intraepithelial neoplasia of cervix}

Geunyoung Kim, " Heekyoung Song, Seongeun Bak, Imhyeon Kim, Soo Young Hur

Department of Obstetrics and Gynecology, Seoul St. Mary's Hospital, College of Medicine, The Catholic University of Korea, Seoul, Korea (songdeng77@naver.com)

Objective: The aim is to identify the genetic and epigenetic factors of intraepithelial neoplasia atypical squamous cells of undetermined significance (ASC-US) or low-grade squamous intraepithelial lesions (LSIL) with high-risk human papilloma virus (HPV) that lead to cancer.

Methods: We selected the patients who diagnosed ASC-US or LSIL of cytology with high-risk HPV infection and followed up at every 6-months intervals. According to the final cytology and HPV test results, the patient group was divided into regression, persistence, and progression and analyzed. DNA extracted from initial and final cytology and matching normal DNA (peripheral blood) were conducted Microsatellite instability (MSI) and MLH1 methylation by real time polymerase chain reaction kit (U-TOP MSI Plus Detection Kit, Epi-TOP mMLH-1 detection Kit, Seasun Biomaterials, Daejeon, Korea). If the sample was insufficient, analyzing of the final sample was omitted. Results: Of 34 patients, 19 patients were regressed their lesion, 10 patients were noted persistent HPV infection. The average age at first diagnosis was $39.8 \pm 10.6$ years. Among 5 patients, their lesions had been progressed and got surgical resection to be removed. The final pathology results were reported highgrade squamous intraepithelial lesions. All 63 cervical cytology samples went through MSI and MLH 1 methylation analysis. Regardless of patients group, all samples were microsatellite stable and negative of MLH1 methylation.

Conclusion: Whether the outcome of HPV infection was progression, persistence or regression, there were no significant difference of MSI, MLH1methylation in DNAs extracted cytology. More studies conducted in tissues other than cytology and studies with expanded number of patients are needed.

\section{Poster (C03)}

Gynecologic Pathology, Genetics and Epidemiology https://doi.org/10.3802/jgo.2021.32.S1.C03

\section{HER2 overexpression and PD-L1 expression in vulvar Paget disease in Thai population: role of potential targeted and immune therapy}

\footnotetext{
Chanya Jiragraivutidej," Ruangsak Lertkhachonsuk, Shanop Shuangshoti, Natkrita Pohthipornthawat

King Chulalongkorn Memorial Hospital, Bangkok, Thailand (por.chanyaa@gmail.com)
}

Objective: To determine the proportion of human 
epidermal growth factor receptor 2 (HER2) overexpression and programmed death-ligand 1 (PD-L1) positive immunohistochemistry in vulvar Paget disease in Thai women. Methods: A cross-sectional study was conducted in King Chulalongkorn Memorial Hospital, Bangkok, Thailand. Histologically diagnosed patients with vulvar Paget disease from January 2000 to April 2020 were identified. Clinical data were collected from medical records and histologic data were verified by pathological review. Pathologic specimens were collected. HER 2 and PD-L1 immunohistochemistry were performed. The results of staining were reported by two pathologists. Data were analyzed using descriptive statistics and reported as percentage and $95 \%$ confidence interval (CI). Clinical outcomes were correlated with immunohistochemistry expression.

Results: Thirty-three vulvar Paget disease cases were included in the study. Twenty patients $(60.6 \%, 95 \% \mathrm{CI}=42.1-77.1)$ had HER2 overexpression. All patients were negative for PD-L1 immunohistochemistry. HER2 positive patients had hazard of death 2.94 (95\% CI=0.61-14.23) times compared with HER2 negative patients. HER2 positive status had relative risk 1.95 and 1.73 for invasive and metastatic disease respectively.

Conclusion: More than half of vulvar Paget disease patients had HER 2 overexpression and might benefit from anti-HER2 targeted therapy. A higher proportion of HER2 overexpression was found in invasive and metastatic vulvar Paget disease. PD-L1 was not expressed in vulvar Paget disease.

Poster (CO4)

Preinvasive Disease of Cervix, Vulva, and Vagina

https://doi.org/10.3802/jgo.2021.32.S1.C04

\section{Is human papillomavirus genotype important in predicting disease progression in women with biopsy-proven negative or CIN1 of LSIL cytology?}

\section{Woodae Kang, * UChul Ju, SeokMo Kim \\ Chonnam National University Medical School, Gwangju, Korea (woodai75@naver.com)}

Objective: Our aim was to estimate the risk of disease incidence in women with low-grade squamous intraepithelial lesion (LSIL) without histology-proven cervical intraepithelial neoplasia grade 2 or worse (CIN2+) by human papillomavirus (HPV) genotype. Methods: Between January 2002 and December 2010, incidence of CIN2+ in 1,580 women including 575 with LSIL and histologyproven negative and 1,005 with LSIL with histology-proven CIN1 was investigated. Baseline high-risk-HPV status was determined by the hybrid capture II assay and high-risk-HPV genotype by the HPV DNA chip test. Cumulative incidence and hazard ratios (HRs) were estimated to explore differences between index data and associations with CIN2+. Results: Of the 1,580 women, 139 (8.8\%) patients developed CIN2+. The 5-year cumulative incidence rate of CIN2+ in HPV-16, HPV-18, and HPV-58 were $18.7 \%, 16.1 \%$, and $12.9 \%$, respectively. On multivariate analysis, being positive in HPV-16 $(\mathrm{HR}=2.324 ; \mathrm{p}<0.01), \mathrm{HPV}-18$ (HR=2.315; $\mathrm{p}<0.01)$, and HPV58 ( $\mathrm{HR}=2.051 ; \mathrm{p}<0.01)$ were significantly associated with developing CIN2+ compared to being negative for that type. Conclusion: Among women with LSIL, HPV-16, HPV-18, or HPV58 positive women may need intensified follow-up as they have the highest risk of becoming CIN2+.

Poster (C05)

Gynecologic Cancer Screening

https://doi.org/10.3802/jgo.2021.32.S1.C05

\section{Liquid-based cytology for cervical cancer screening compared between spatula-cotton swab and spatula-cytobrush: a randomized controlled trial}

\section{Perapong Inthasorn," Wiphawee Hochaame, Somsak Laiwejpithaya, Chatchai Areeswate \\ Siriraj Hospital, Bangkok, Thailand (perapong_i@hotmail.com)}

Objective: The presence of endocervical cell/transformation zone $(\mathrm{EC} / \mathrm{TZ})$ component is still described as a quality indicator in the 2014 Bethesda System. In addition, the presence or absence of $\mathrm{EC} / \mathrm{TZ}$ component is a useful quality assurance measurement. The aim of this study was to compare the percentage of satisfactory samples with presence of EC/TZ components between samples collected by spatula-cotton swab and by spatula-cytobrush for cervical cancer screening using Siriraj liquid-based cytology.

Methods: This randomized controlled trial was conducted during January 2020-March 2020. A total of 1,400 women were recruited. Study women were randomly allocated to either the spatula-cotton swab group or the spatula-cytobrush group (700 women per group). All samples were sent for cytologic interpretation and determination of satisfactory sample with presence of EC/TZ component.

Results: There were no significant differences in baseline characteristics between groups. The percentage of satisfactory samples with presence of EC/TZ components was significantly higher in the spatula-cytobrush group than in the spatulacotton swab group $(87.1 \%$ vs. $75.9 \%$, respectively, $\mathrm{p}<0.001)$. There were no significant differences between 2 groups relative 